\title{
Dynamic Determinacy and the Existence of Sunspot Equilibria*
}

\author{
JOHN LAITNER \\ Department of Economies, Lorch Hall, The University of Michigan, \\ Ann Arbor, Michigan 48109
}

Received May 22, 1986; revised February 10, 1988

\begin{abstract}
This paper relates the existence of stationary sunspot equilibria in the vicinity of a conventional stationary state to the phase diagram surrounding the latter. We find that the local condition required for a unique equilibrium path returning to the stationary state following any slight disturbance of initial conditions is also sufficient to exclude stationary sunspot equilibria in the stationary state's immediate vicinity. In that sense, an eigenvalue condition familiar from dynamic analysis can tell us something about the possibilities of local sunspot activity as well. Journal of Economic Literature Classification Numbers: 022, 023, 111. (C) 1989 Academic Press, Inc.
\end{abstract}

If we can think of an economic model as defining a function from agents preferences, endowments, and technologies to market prices and quantities, comparative statics exercises will be straightforward. Recent work reveals two potential complications, however: the possibility of large numbers of sunspot solutions, even arbitrarily close to more conventional outcomes; and indeterminacy. In the former instance, an "extrinsic random variable"- $a$ variable with realizations not directly affecting the underlying characteristics of households or production-can influence equilibrium outcomes. Essentially, if all agents think a sunspot variable is important, it may become so-see, for example, Shell [16], Cass and Shell [6], and Azariadis [1]. In the case of indeterminacy, a model (generically) may exhibit a continuum of equilibria (unrelated to extrinsic randomness) consistent with given initial conditions, perfect foresight, and market clearing restrictions. In fact, a stationary solution may have a continuum of equilibrium paths in its local vicinity converging to it-see, for instance, Calvo [7], Laitner [13], Woodford [18], and Kehoe and Levine [12]. In either case, we are left unsure about which equilibrium a given model should direct us to after a parameter change.

* I owe thanks for very helpful comments to this journal's referees and to $K$. Shell. This research was supported by the National Science Foundation, Grant SES-8106555. 
The purpose of this paper is to show that a condition needed for avoiding indeterminacy in the vicinity of a stationary solution, say, $x^{*}$, also rules out stationary sunspot equilibria arbitrarily close to $x^{*}$. Thus, an eigenvalue condition characterizing local (equilibrium) dynamics also yields results about sunspots. More precisely, if a model with no historical (or "predetermined") endogenous variables has a stationary solution $x^{*}$ which is "determinate" in the sense that every possible given initial condition in some open neighborhood of it constitutes the starting point of a single cquilibrium timc path which converges to it, then Theorem 1 below establishes that for any (finite) integer $M$ there exists an open neighborhood of $x^{*}$ not containing (entirely within it) a stationary sunspot equilibrium based on any extrinsic random variable having $M$ states. Theorem 3 presents identical results for models containing historical as well as non-historical arguments. Our framework allows an arbitrary (finite) number of state variables.

Our theorems complement a number of existing articles. For example, Azariadis [1], Azariadis and Guesnerie [2], Guesnerie [11], and Peck [15, Theorem 2a] examine, in the context of consumption loan frameworks with no historical variables, the sufficiency for the existence of stationary sunspot equilibria of having one or more eigenvalues of modulus less than 1 for a model's forward dynamics in the vicinity of a conventional stationary solution-in fact, a condition leading to indeterminacy (see Section 1). Woodford [19] considers a model with infinite lived agents. The local dynamics can be analyzed in terms of two state variables-one historical and the other nonhistorical. He derives a similar result-showing that having two stable eigenvalues, which implies dynamic indeterminacy, is sufficient for the existence of a stationary sunspot in each neighborhood of a given stationary solution. Grandmont $[9,10]$ obtains results for both existence and non-existence: for a consumption loan model having a single non-historical state variable in each period, he proves that an eigenvalue condition implying local instability in the backward dynamics (in other words, the case with indeterminacy-see Section 1) is necessary and sufficient for local sunspot activity. Woodford [18] summarizes a large number of examples in the same vein. ${ }^{1}$

The macroeconomics literature on linear rational expectations models (with intrinsic randomness) provides parallel results. In particular, Blanchard and Kahn [4] show that eigenvalue configurations leading to dynamic indeterminacy imply the existence of solutions containing extrinsic random variables (see also Gourieroux, Laffont, and Monfort [8] and Broze, Gourieroux, and Szafarz [5]).

\footnotetext{
${ }^{1}$ As revisions on this paper proceeded, the author became aware of Woodford [20], which independently (and with a somewhat different technology) reaches conclusions analogous to ours.
} 
The organization of the present paper is as follows. Section 1 sets up our framework of analysis. Section 2 introduces sunspots and presents our theorems, and Sections 3, 4 provide proofs.

\section{Local Dynamics}

Until the introduction of extrinsic uncertainty, the model we consider consists of the following elements: a time- $t$ vector of state variables $x_{t} \in \mathbb{R}^{n}$, a (time-autonomous) system of excess demand functions $Z\left(x_{t}, x_{t+1}\right) \in \mathbb{R}^{n}$, and a set of initial conditions. The vector $x_{t}$ may contain prices and/or quantity variables such as capital stock figures. Time is discrete. We restrict our atention to equilibrium time paths.

Our analysis begins at time 0 . The sequence of vectors $\left\{x_{0}, x_{1}, \ldots\right\}$ is an "equilibrium" if $Z\left(x_{t}, x_{t+1}\right)=0$ all $t \geqslant 0$ and if $x_{0}$ satisfies initial conditions. This definition implicitly requires perfect foresight. The vector $x^{*} \in R^{n}$ defines a "stationary equilibrium" if $Z\left(x^{*}, x^{*}\right)=0$. Assume such an $x^{*}$ exists. Normalize variables so that $x^{*}=0$.

To consider non-stationary equilibrium paths in the vicinity of $x^{*}$, assume that $Z(\cdot, \cdot)$ is twice continuously differentiable in some open neighborhood of $\left(x^{*}, x^{*}\right)$. Define

$$
A_{1} \equiv \partial Z\left(x^{*}, x^{*}\right) / \partial x_{t}, \quad A_{2} \equiv \partial Z\left(x^{*}, x^{*}\right) / \partial x_{t+1} .
$$

Assume

$$
\operatorname{det}\left(A_{2}\right) \neq 0 \text {. }
$$

Using the implicit function theorem and $(\mathrm{A} 1), Z(\cdot, \cdot)$ uniquely defines a difference equation

$$
x_{t+1}=\zeta\left(x_{t}\right) \quad \text { all } \quad t \geqslant 0
$$

with

$$
Z\left(x_{t}, \zeta\left(x_{\varepsilon}\right)\right)=0
$$

all $x_{i}$ in some open neighborhood of $x^{*}$. Well-known theorems show we can study the behavior of (1) locally using the linear system

$$
x_{t+1}=A \cdot x_{t} \quad \text { with } A \equiv-\left(A_{2}\right)^{-1} \cdot A_{1} .
$$

For simplicity, we assume below that the eigenvalues of $A$ are distinct and that none have modulus 1 .

Consider the makeup of the vector $x_{t}=\left(x_{1 t}, \ldots, x_{n t}\right)$. Some components may be "historical" variables: variables with time- $t$ levels fixed by events of 
prior dates (for example, lags may mean the time- $t$ physical capital stock depends only on earlier investment behavior). The remaining elements of $x_{t}$ are "non-historical" - their values being determined during period $t$. Without loss of generality let $x_{i t}, i=1, \ldots, h$, be historical, $u_{t} \equiv\left(x_{1 t}, \ldots, x_{h t}\right)$, and $v_{t} \equiv\left(x_{h+1, t}, \ldots, x_{n t}\right)$. Then given initial conditions will consist only of values for $u_{0}$; our analysis must determine $v_{0}$ and $x_{t}=\left(u_{t}, v_{t}\right)$ all $t>0$.

If $h^{*}$ eigenvalues of $A$ have modulus less than 1, consider three cases: (i) $h^{*}<h$; (ii) $h^{*}=h$; and (iii) $h^{*}>h$. Stationary equilibria have, of course, long been a cornerstone of dynamic analysis. For $x^{*}$ to warrant special attention, however, we presumably want to insist on being able to reach it (via an equilibrium time path) from any $u_{0}$ in an open neighborhood of $0 \in R^{h}$-ruling out, in general, case (i). In case (iii), for any $u_{0}$ in a small enough open set containing $0 \in R^{h}$ there will be a continuum of values $v_{0} \in R^{n-h}$ such that $x_{t+1}=\zeta\left(x_{t}\right)$ all $t \geqslant 0$ implies $\left|x_{t}-x^{*}\right| \rightarrow 0$ as $t \rightarrow \infty$. This is the "indeterminate" case. For the possibility of determinacy within the class of convergent equilibrium paths, we are left with only case (ii). ${ }^{2}$ Laitner [13], Kehoe and Levine [12], and Begg [3, Chap. 3] discuss similar issues.

A precise condition for a unique convergent equilibrium path originating from each $x_{0}=\left(u_{0}, v_{0}\right)$ with $u_{0}$ in some open neighborhood $\mathscr{U}$ of $u^{*}$ (where $\left.x^{*} \equiv\left(u^{*}, v^{*}\right)\right)$ and lying entirely within some small given open set $\mathscr{V}$ containing $x^{*}$ is

Condition 1. The matrix $A$ has $h$ distinct eigenvalues $e_{t}$ with $\left|e_{t}\right|<1$ and $n-h$ distinct eigenvalues $e_{j}$ with $\left|e_{j}\right|>1$. The projection of the stable manifold for (2) onto the space containing all historical subvectors $u$ has dimension $h$.

\section{SUNSPOT EQUILIBRIA}

Suppose we have an extrinsic random variable with outcomes $a_{1}, \ldots, a_{M}$. As stated, "extrinsic" means the variable's realizations have no affect on agents' preferences, on production technologies, or on endowments. Let the random variable have transition matrix $\Pi=\left[\pi_{i j}\right]$-the probability of transiting from state $a_{i}$ at time $t$ to state $a_{j}$ at time $t+1$ being $\pi_{i j}$. Assume all agents in the model can observe the current realization $a_{i}$ and know $\Pi$. We now define a stationary sunspot equilibrium and present our theorems.

Following Guesnerie [11], if all agents anticipate time- $(t+1)$ state vector $x_{t+1}\left(a_{j}\right)$ conditional on $a_{j}$ (in that time period), if all maximize

\footnotetext{
${ }^{2}$ Nonconvergent paths may lead to contradictions of market clearing conditions within a finite number of periods-or they may define legitimate equilibria (see, for example, Woodford [18]).
} 
expected utility, and if we have outcome $a_{i}$ at time $t$, let time- $t$ excess demand be

$$
\tilde{Z}\left(x_{t}\left(a_{i}\right), x_{t+1}\left(a_{1}\right), \ldots, x_{t+1}\left(a_{M}\right) ; \Pi\right) \in R^{n} .
$$

Our definition of a stationary sunspot equilibrium is conventional:

Definition 1. For any $\infty>M \geqslant 2$, the vectors $x\left(a_{1}\right), \ldots, x\left(a_{M}\right) \in R^{n}$, at least two of which differ from one another, and the $n \times n$ probability transition matrix $I I$ for the extrinsic random variable having realizations $a_{1}, \ldots, a_{M}$ determine a "stationary $M$-sunspot equilibrium" if for all $i=1, \ldots, M, \tilde{Z}\left(x\left(a_{i}\right), x\left(a_{1}\right), \ldots, x\left(a_{M}\right) ; \Pi\right)=0$.

Thus, $x\left(a_{1}\right), \ldots, x\left(a_{M}\right)$ and $I I$ characterize a stationary sunspot equilibrium if given a time- $t$ realization of the extrinsic variable $a_{i}, x_{t}=x\left(a_{i}\right)$ clears all current markets provided agents anticipate $x_{t+1}=x\left(a_{j}\right)$ in the event of random realization $a_{j}$ next period.

Assume $\tilde{Z}(\cdot)$ is twice continuously differentiable. In the special instance with

$$
x_{t+1}\left(a_{1}\right)=x_{t+1}\left(a_{2}\right)=\cdots=x_{t+1}\left(a_{M}\right) \equiv \bar{x},
$$

for any $x$ we should have

$$
\tilde{Z}(x, \bar{x}, \ldots, \bar{x} ; \Pi)=Z(x, \bar{x}) .
$$

Two derivative properties follow from the underlying model's first-order conditions in the same special case: for any $\Pi$,

$$
\begin{aligned}
\tilde{Z}_{1}(x, \bar{x}, \ldots, \bar{x} ; \Pi) & =Z_{1}(x, \bar{x}), \\
\tilde{Z}_{j+1}(x, \bar{x}, \ldots, \bar{x} ; \Pi) & =\pi_{i j} \cdot Z_{2}(x, \bar{x}) \quad \forall j=1, \ldots, M .
\end{aligned}
$$

We treat these as assumptions-- see Guesnerie [11, p. 108].

Let $\|\cdot\|$ be the Euclidean norm. Fix any $M<\infty$. Define the "radius" of any $M$-sunspot equilibrium characterized by

$$
s \equiv\left(x\left(a_{1}\right), \ldots, x\left(a_{M}\right), \Pi\right)
$$

as

$$
r(s) \equiv \sum_{j=1}^{M}\left\|x\left(a_{j}\right)\right\|
$$

Our first result is

THEOREM 1. Suppose $h=0$-so that a model's endogenous variables are all non-historical. Suppose assumptions (A1)-(A4) and Condition 1 hold for 
stationary state $x^{*}=0$. Fix any $M<\infty$. Then there exists $\varepsilon_{M}>0$ such that no stationary $M$-sunspot equilibrium has radius less than $\varepsilon_{M}$.

Section 1 explains that Condition 1 leads to the saddlepoint configuration in the neighborhood of $x^{*}$ desired for a unique convergent equilibrium time path. Theorem 1 shows that in the context of a model with no predetermined variables-such as the popular consumption loan systems mentioned in the introduction-for any $M$, the same saddlepoint implies the existence of an open set $\mathscr{V}_{M}$ containing $x^{*}$ such that no stationary $M$-sunspot equilibrium exists having all state vectors, $x\left(a_{i}\right)$, in $\mathscr{V}_{M}$. In the latter particular sense Condition 1 ensures that $x^{*}$ is "isolated" from sunspot equilibria. ${ }^{3}$

Section 3 presents a formal proof of Theorem 1. The idea, however, is as follows. We can approximate the equilibrium behavior of our model in the local vicinity of $x^{*}$ with a linearized system. When $h=0$, determinacy requires that all eigenvalues of our model have moduli exceeding 1 -in other words, the linear version explodes from any initial vector $x_{0} \neq x^{*}$. In the case of a sunspot equilibrium, we replace $x_{1}$ with a set of vectors- $x_{1}\left(a_{1}\right), \ldots, x_{1}\left(a_{M}\right)$-each assigned a probability weight. In the linearized microcosm near $x^{*}$ - given (A2)-(A4)-the model associates $A \cdot x_{0}$ (see line (2)) with the mean $x_{1}$. Similarly, the model associates $A \cdot x_{i}\left(a_{i}\right)$, for any realization $a_{i}$, with a mean outcome for $x_{2}$, etc. An explosive $A$ thrusts the mean away from $x^{*}$--which is not compatible with "stationarity" of the sunspot equilibrium.

The same result holds when the model has historical as well as nonhistorical variables. Before stating the new theorem-Theorem 3-however, we need the connecting link of Theorem 2 .

For a model with only historical variables, sunspot equilibria are impossible: if all the components of state variable $x$ are historical $\left(x_{t}=u_{t}\right)$, then all the elements of $x_{t+1}$ are determined by period $t$; hence, at time $t$ agents cannot rationally anticipate several distinct outcomes for $x_{t+1}$ related to intrinsic sunspot realizations occurring at $t+1 .{ }^{4}$ Similarly, if the model has a combination of historical and non-historical variables, agents at time $t$ can anticipate sunspot variations only in the elements $v_{t+1}$ of $x_{t+1}$.

Formalizing this observation,

THEOREM 2. Let $\left(x\left(a_{1}\right), \ldots, x\left(a_{M}\right), \Pi\right)$ determine a stationary $M$-sunspot equilibrium. Let $\Pi=\left[\pi_{i j}\right]$. Suppose $x\left(a_{i}\right) \equiv\left(u\left(a_{i}\right), v\left(a_{i}\right)\right)$ with $u\left(a_{i}\right) \in R^{h}$ being

\footnotetext{
${ }^{3}$ The theorem does not rule out sunspots with some, but not all, state vectors arbitrarily close to $x^{*}$-see, especially, Grandmont $[10$, p. 23$]$.

${ }^{4}$ So, for models with no non-historical variables, for example, the standard Solow [17] model, sunspot equilibria are never an issue.
} 
a subvector of historical variables. Then for any $i=1, \ldots, M$, there must be $u_{i} \in R^{h}$ such that for every $j=1, \ldots, M$ with $\pi_{i j}>0$, the subvector $u\left(a_{j}\right)=u_{i}$.

Now we can proceed to

THEOREM 3. Suppose $h>0$. Suppose assumptions (A1)-(A4) and Condition 1 hold for stationary state $x^{*}=0$. Fix any $M<\infty$. Then there exists an $\varepsilon_{M}>0$ such that no stationary $M$-sunspot equilibrium $s$ has radius less than $\varepsilon_{M}$.

As before, the theorem shows that if we have the phase diagram needed for determinacy, for any $M$ we can find an open neighborhood $\mathscr{V}_{M}$ of $x^{*}$ such that no stationary $M$-sunspot equilibrium exists having all state vectors in $\mathscr{V}_{M}$.

Section 4 presents a proof. The idea is to combine Theorems 1 and 2. Suppose we start at $x_{0} \neq x^{*}$ and restrict attention to the vicinity of $x^{*}$. In the sunspot case, there will be a set of outcomes for $x_{1}-x_{1}\left(a_{1}\right), \ldots, x_{1}\left(a_{M}\right)$ each with a probability weight. Given a realization $a_{i}$, we apply the model to $x_{1}\left(a_{i}\right)$ to derive the set for $x_{2}$, etc. For the sunspot equilibrium to be stationary, roughly speaking, we must avoid letting $A$-see line (2)-make $x_{t}$ explode. That entails having $x_{t}\left(a_{i}\right)$ all $t$ and $i$ in the stable manifold for $A$-implying, in turn, a set of $n-h$ linear constraints for each $x_{t}\left(a_{i}\right)$. Theorem 2 implies $h$ more constraints. Thus, given $x_{t}$, the $h$ plus $n-h$ constraints fully (hence, uniquely) determine $x_{t+1}$-allowing us to write $x_{t+1}=A \cdot x_{t}$. Finally, repeated multiplication by $A$ within the stable manifold takes us to the origin; thus, the only sunspot very close to $x^{*}$ is the degenerate one consisting of permanent repetitions of $x^{*}$ itself.

\section{Proof of Theorem 1}

The proof of Theorem 1 is straightforward. We begin with a preliminary lemma.

Fix any $M$. Suppose each open neighborhood $\mathscr{V}$ of $x^{*}$ contains a stationary $M$-sunspot equilibrium. Then for each $k=1,2, \ldots$ we can find a stationary $M$-sunspot equilibrium, characterized by $s_{k} \equiv\left(x_{k}\left(a_{1}\right), \ldots\right.$, $\left.x_{k}\left(a_{M}\right), \Pi_{k}\right)$, with

$$
r\left(s_{k}\right) \leqslant 1 / k
$$

Define

$$
X_{k}\left(a_{i}\right) \equiv x_{k}\left(a_{i}\right) / \sum_{j=1}^{M}\left\|x_{k}\left(a_{j}\right)\right\| .
$$

${ }^{5}$ Note that $(\mathrm{A} 2)$ implies the derivatives of $\tilde{Z}\left(x^{*}, x^{*}, \ldots, x^{*}, \Pi^{*}\right)$ with respect to the elements of $\Pi^{*}$ are all 0 . 
(Definition 1 shows we are not dividing by 0 .) Then since $\left(X_{k}\left(a_{1}\right), \ldots\right.$, $\left.X_{k}\left(a_{M}\right)\right)$ for each $k$ lies in the compact set

$$
\mathscr{S} \equiv\left\{\left(X_{1}, \ldots, X_{M}\right) \mid \text { for all } i, X_{i} \in R^{n} \text { and } \sum_{j=1}^{M}\left\|X_{j}\right\|=1\right\} \subset R^{n \cdot M},
$$

and since each row of $\Pi_{k}$ lies in the unit simplex in $R^{M}$, there is a vector

$$
\left(X^{*}\left(a_{1}\right), \ldots, X^{*}\left(a_{M}\right)\right) \in \mathscr{S},
$$

a probability transition matrix $\Pi^{*}$, and a subsequence

$$
S_{k(l)} \equiv\left\{\left(X_{k(l)}\left(a_{1}\right), \ldots, X_{k(l)}\left(a_{M}\right), \Pi_{k(l)}\right\} \quad \text { all } \quad l=1,2, \ldots,\right.
$$

with

$$
\lim _{l \rightarrow \infty} S_{k(l)}=\left(X^{*}(a), \ldots, X^{*}\left(a_{M}\right), \Pi^{*}\right) .
$$

We now use a Taylor series approximation. Let $\tilde{Z}_{i}(\cdot)$ be the derivative of $\tilde{Z}(\cdot)$ with respect to its $i$ th (vector) argument. Using linear approximations and (A2), ${ }^{5}$

$$
\begin{gathered}
\tilde{Z}_{1}\left(x^{*}, x^{*}, \ldots, x^{*}, \Pi^{*}\right) \cdot x_{k}\left(a_{i}\right)+\sum_{j=1}^{M} \tilde{Z}_{j+1}\left(x^{*}, x^{*}, \ldots, x^{*}, \Pi^{*}\right) \cdot x_{k}\left(a_{j}\right) \\
\approx \tilde{Z}\left(x_{k}\left(a_{i}\right), x_{k}\left(a_{1}\right), \ldots, x_{k}\left(a_{M}\right) ; \Pi_{k}\right)=0 \quad \forall i=1, \ldots, M .
\end{gathered}
$$

The error is second order. Thus, dividing by $\sum_{j=1}^{M}\left\|x_{k}\left(a_{j}\right)\right\|$ and taking limits as in (8) (using subsequences if necessary),

$$
\begin{gathered}
\tilde{Z}_{1}\left(x^{*}, x^{*}, \ldots, x^{*}, \Pi^{*}\right) \cdot X^{*}\left(a_{i}\right)+\sum_{j=1}^{M} \tilde{Z}_{j+1}\left(x^{*}, x^{*}, \ldots, x^{*}, \Pi^{*}\right) \\
\times X^{*}\left(a_{j}\right)=0 \quad \forall i=1, \ldots, M .
\end{gathered}
$$

Employing (A3) and (A4) and the notation of line (2), (10) becomes

$$
A_{1} \cdot X^{*}\left(a_{i}\right)+\sum_{j=1}^{M} \pi_{i j}^{*} \cdot A_{2} \cdot X^{*}\left(a_{j}\right)=0 \quad \forall i=1, \ldots, M,
$$

where $\Pi^{*} \equiv\left[\pi_{i j}^{*}\right]$. Using (A1) and (11),

$$
A \cdot X^{*}\left(a_{i}\right)=\sum_{j=1}^{M} \pi_{i j}^{*} \cdot X^{*}\left(a_{j}\right) \quad \forall i
$$

We can use (12) to show that $X^{*}\left(a_{i}\right)$ all $i=1, \ldots, M$ must lie in the stable manifold for (2). 
Lemma 1: Let $X^{*}\left(a_{i}\right)$ all $i=1, \ldots, M$ be as above. Let (A1)-(A4) hold. Then each $X^{*}\left(a_{i}\right)$ lies in the stable manifold for (2).

Proof. Define $\left(\Pi^{*}\right)^{m}=\left[\pi_{i j}^{*}(m)\right]$ all $m=1, \ldots$. Multiplying (12) through by $A$,

$$
\begin{aligned}
A^{2} \cdot X^{*}\left(a_{i}\right) & =\sum_{j=1}^{M} \pi_{i j}^{*} \cdot A \cdot X^{*}\left(a_{j}\right) \\
& =\sum_{j=1}^{M} \pi_{i j}^{*} \cdot \sum_{l=1}^{M} \pi_{j l}^{*} \cdot X^{*}\left(a_{l}\right) \\
& =\sum_{i=1}^{M}\left(\sum_{j=1}^{M} \pi_{i j}^{*} \cdot \pi_{j i}^{*}\right) \cdot X^{*}\left(a_{l}\right) \\
& =\sum_{l=1}^{M} \pi_{i l}^{*}(2) \cdot X^{*}\left(a_{l}\right) \quad \forall i=1, \ldots, M .
\end{aligned}
$$

Repeating the process, for any $m=2,3, \ldots$,

$$
A^{m} \cdot X^{*}\left(a_{i}\right)=\sum_{j=1}^{M} \pi_{i j}^{*}(m) \cdot X^{*}\left(a_{j}\right) \quad \forall i=1, \ldots, M .
$$

Now $\left(I^{*}\right)^{m}$ is itself a probability transition matrix. Thus, for any $m$,

$$
\begin{aligned}
\left\|\sum_{j=1}^{M} \pi_{i j}^{*}(m) \cdot X^{*}\left(a_{j}\right)\right\| & \leqslant \sum_{j=1}^{M} \pi_{i j}^{*}(m) \cdot\left\|X^{*}\left(a_{j}\right)\right\| \\
& \leqslant \sum_{j=1}^{M}\left\|X^{*}\left(a_{j}\right)\right\|=1 .
\end{aligned}
$$

If $X^{*}\left(a_{i}\right)$ is not in the stable manifold for (2), some component of the vector on the left-hand side of (13) must diverge for large enough $m$ - contradicting (14).

Q.E.D.

In words, if $X_{t}=X^{*}\left(a_{i}\right)$, the right-hand side of (12) gives the expected values (as of time $t$ ) for $X_{t+1}$. Multiplying through by $A$, we generate the expected value (as of time $t$ ) for $X_{t+2}$. Continuing in this way, if we began off of the stable manifold for (2), the expected values must explode-an impossibility for $\left(X^{*}\left(a_{1}\right), \ldots, X^{*}\left(a_{M}\right)\right)$ derived from "stationary" sunspot equilibria. This result generalizes Guesnerie [11, Theorem 3, part 2].

The proof of Theorem 1 follows immediately:

Proof of Theorem 1. Fix $M$. Suppose each open neighborhood $\mathscr{W}$ of $x^{*}$ contains a stationary $M$-sunspot equilibrium. Construct $X^{*}\left(a_{i}\right)$ all $i=1, \ldots, M$ as above. Since $h=0$, the stable manifold for $(2)$ is only the origin. Thus, Lemma 1 implies $X^{*}\left(a_{i}\right)=0 \in R^{n}$ all $i$. But, by construction, $\left(X^{*}\left(a_{1}\right), \ldots, X^{*}\left(a_{M}\right)\right) \in \mathscr{P}$, a contradiction since $0 \notin \mathscr{S}$.

Q.E.D. 


\section{Proof of Theorem 3}

The idea of the proof of Theorem 3 is given in Section 2. More formally, suppose that Condition 1 holds and that we have a stationary $M$-sunspot equilibrium in every open neighborhood $\mathscr{V}$ of $x^{*}$. Construct a convergent subsesquence as in Section 3. If $\pi_{i j}^{*}>0$ and $\pi_{i l}^{*}>0$, the argument of Theorem 2 shows that $X^{*}\left(a_{j}\right)$ and $X^{*}\left(a_{l}\right)$ must have common historical components. Lemma 1 shows they must both be in the stable manifold for (2). Given the last part of Condition 1, we can then see that $X^{*}\left(a_{j}\right)=$ $X^{*}\left(a_{i}\right)$. Thus, (12) collapses to

$$
A \cdot X^{*}\left(a_{i}\right)=X^{*}\left(a_{j}\right) .
$$

The same reasoning applies for all $i$. With each $X^{*}(\cdot)$ on the stable manifold for (2), however, such multiplication leads to convergence to 0 for all states which are not transitory (under $\Pi^{*}$ ). Furthermore, every state cannot be transitory.

We need an additional definition. For $m=1,2, \ldots$, use the notation

$$
(\Pi)^{m}=\Pi \cdot \Pi \cdot \ldots \cdot \Pi \equiv\left[\pi_{i j}(m)\right] .
$$

Then define

$$
p(i, T, \Pi) \equiv \sum_{m=1}^{T} \pi_{i i}(m)
$$

If we have a stationary $M$-sunspot equilibrium $s$ (see line (3)), and if the extrinsic random variable's current realization is $a_{i}, p(i, T, \Pi)$ gives the probability of at least one repeat of $a_{i}$ over the next $T$ periods. If the realization $a_{i}$ is visited very infrequently, $p(i, T, s)$ will be near 0 even for a large $T$. If $a_{i}$ is actually a transitory state,

$$
\lim _{T \rightarrow \infty} p(i, T, \Pi)=0 .
$$

We now present our proof.

Proof of Theorem 3. Fix any $M<\infty$. Suppose (A1)-(A4) and Condition 1 hold. If there exists some open neighborhood of $x^{*}=0$ not containing any stationary $M$-sunspot equilibrium, we are done. Otherwise, for any $k=1,2, \ldots$ we can find a stationary $M$-sunspot equilibrium

$$
s_{k} \equiv\left(x_{k}\left(a_{1}\right), \ldots, x_{k}\left(a_{M}\right), \Pi_{k}\right)
$$

with $r\left(s_{k}\right)<1 / k$. Define the corresponding $S_{k}$ as in Section 3, with, for some subsequence $S_{k(l)}$,

$$
\lim _{l \rightarrow \infty} S_{k(l)} \equiv S^{*} \equiv\left(X^{*}\left(a_{1}\right), \ldots, X^{*}\left(a_{M}\right), \Pi^{*}\right) .
$$


Consider any $i=1, \ldots, M$. Let $\Pi_{k} \equiv\left[\pi_{i j}^{k}\right]$ be the transition matrix in $s_{k}$ (and $S_{k}$ ). Let $I^{*} \equiv\left[\pi_{i j}^{*}\right]$. If $\pi_{i j}^{*}>0$ and $\pi_{i l}^{*}>0$, by the definition of $\Pi^{*}$ we can find $L$ so large that $l^{*}>L$ implics $\pi_{i j}^{k\left(l^{*}\right)}>0$ and $\pi_{i l}^{k\left(l^{*}\right)}>0$. Let $x_{k}\left(a_{m}\right)=\left(u_{k}\left(a_{m}\right), v_{k}\left(a_{m}\right)\right)$. Then Theorem 2 implies there exists $u_{i}$ such that $u_{k\left(l^{*}\right)}\left(a_{j}\right)=u_{i}=u_{k\left(l^{*}\right)}\left(a_{i}\right)$ all $l^{*} \geqslant L$. Let $X^{*}\left(a_{m}\right)=\left(U^{*}\left(a_{m}\right), V^{*}\left(a_{m}\right)\right)$. Then by construction, $U^{*}\left(a_{j}\right)=U^{*}\left(a_{l}\right)$.

Lemma 1 shows $X^{*}\left(a_{m}\right)$ is in the stable manifold for (2) each $m=1, \ldots, M$. Hence, the preceding paragraph and the last part of Condition 1 imply $X^{*}\left(a_{j}\right)=X^{*}\left(a_{l}\right)$ when $\pi_{i j}^{*}>0$ and $\pi_{i l}^{*}>0$ (see the discussion in the text). Hence, for any $i$, if $\pi_{i j}^{*}>0$,

$$
A \cdot X^{*}\left(a_{i}\right)=X^{*}\left(a_{j}\right)
$$

Repeating, if $\pi_{j i}^{*}>0$,

$$
A^{2} \cdot X^{*}\left(a_{i}\right)=X^{*}\left(a_{l}\right)
$$

Pick some $i$. Suppose that $p\left(i, T, \Pi^{*}\right)>0$ some $T$. Then $\pi_{i i}^{*}(m)>0$ some $m \in\{1, \ldots, T\}$. Thus, going back to the preceding paragraph,

$$
A^{m} \cdot X^{*}\left(a_{i}\right)=X^{*}\left(a_{i}\right)
$$

Multiply by $A^{m}$ over and over, and using (15), for any $v=1,2, \ldots$,

$$
A^{m \cdot v} \cdot X^{*}\left(a_{i}\right)=X^{*}\left(a_{i}\right) \text {. }
$$

So, using Lemma 1 ,

$$
X^{*}\left(a_{i}\right)=\lim _{v \rightarrow \infty} A^{m \cdot v} \cdot X^{*}\left(a_{i}\right)=0
$$

Hence, for any $i-1, \ldots, M$,

$$
p\left(i, T, \Pi^{*}\right)=0 \text { all } T, \quad \text { or } \quad X^{*}\left(a_{i}\right)=0 .
$$

The latter is impossible because $X^{*}\left(a_{i}\right) \in \mathscr{I}$ by construction, and $S$ does not include the origin. On the other hand, $p\left(i, T, \Pi^{*}\right)=0$ all $T$ implies $i$ is a transitory state. Since not all states $i$ can be transitory, we have a contradiction.

Q.E.D.

\section{CONCLUSION}

Thcorcms 1 and 2 cstablish a relationship between determinacy and the existence of sunspot equilibria. This enhances the value of learning about a given stationary state's (local) determinacy (see, for example, 
[14])-although it unfortunately seems to have little bearing on the existence of stationary sunspot equilibria with at least some state outcomes isolated from $x^{*}$.

\section{REFERENCES}

1. C. Azariadis, Self-fulfilling prophecies, J. Econ. Theory 25 (1981), 380-396.

2. C. Azariadis and R. Guesnerie, Sunspots and cycles, Rev. Econ. Stud. 53 (1986), $725-738$.

3. D. BEGG, "The Rational Expectations Revolution in Macroeconomics," The Johns Hopkins Univ. Press, Baltimore, MD, 1982.

4. O. BLANCHARD AND C. KAHN, The solution of linear difference models under rational expectations, Econometrica 48 (1980), 1305-1311.

5. L. Broze, C. Gourieroux, and A. Szafarz, Solutions of linear rational expectations models, Econometrica Theory 1 (1985), 315-340.

6. D. CASS AND K. Shell, Do sunspots matter? J. Polit. Econ. 91 (1983), 193-227.

7. G. CALvo, On the indeterminacy of interest rates and wages with perfect foresight, J. Econ. Theory 19 (1978), 321-337.

8. C. Gourieroux, J. LAFFont, And A. Monfort, Rational expectations in dynamic linear models: Analysis of solutions, Econometrica 50 (1982), 409-426.

9. J. Grandmont, Stabilizing competitive business cycles, J. Econ. Theory 40 (1986), 57-76.

10. J. Grandmont, Local bifurcations and stationary sunspots (Part I), CEPREMAP and CNRS, Paris, mimeo, 1986.

11. R. GUESNERIE, Stationary sunspot equilibria in an $N$ commodity world, $J$. Econ. Theory 40 (1986), 103-127.

12. T. Kehoe AND D. LeVINE, Comparative statics and perfect foresight in infinite horizon economies, Econometrica 53 (1985), 433-454.

13. J. LAITNER, The definition of stability in models with perfect foresight, J. Econ. Theory 28 (1982), 347-353.

14. J. LAITNER, Transition time paths for overlapping-generations models, J. Econ. Dynam. Control 7 (1984), 111-129.

15. J. PECK, On the existence of sunspot equilibria in an overlapping generations model, $J$. Econ. Theory 44 (1988), 19-42.

16. K. SHELL, Monnaie et allocation intertemporelle (text in English, abstract in French), CNRS Seminaire Roy-Malinvaud, Paris, minmeo, 1977.

17. R. Solow, A contribution to the theory of economic growth, Quart. J. Econ. 70 (1956), 65-94.

18. M. WOODFORD, Indeterminacy of equilibrium in the overlapping generations model: A survey, Columbia University, mimeo, 1984.

19. M. Woodford, Stationary sunspot equilibria in a finance constrained economy, I. Econ. Theory 40 (1986), 128-137.

20. M. Woodford, Stationary sunspot equilibria, University of Chicago, mimeo, 1986. 